\title{
Simultaneous Selection is Effective in Increasing Betalain Pigment Concentration but not Total Dissolved Solids in Red Beet
}

\author{
I.L. Goldman', K.A. Eagen ${ }^{2}$, D.N. Breitbach ${ }^{3}$, and W.H. Gabelman ${ }^{4}$ \\ Department of Horticulture, University of Wisconsin-Madison, 1575 Linden Drive, Madison, WI 53706
}

Additional index words. betacyanin, betaxanthin, recurrent selection

\begin{abstract}
Betalain pigments, composed of the red-violet betacyanins (BC) and the yellow betaxanthins (BX), provide an excellent natural alternative to synthetic red dyes. The objective of this investigation was to evaluate eight cycles of recurrent selection in two red beet (Beta vulgaris $\mathrm{L}$.) populations selected for high total betalain pigment concentration and both high (HPHS) and low (HPLS) total dissolved solids (TDS). Remnant seed was planted in a randomized complete block design in replicated trials at two locations in 1994. Beet roots were sampled at harvest for concentrations of BC, BX, and TDS. Highly significant differences among cycles for BC, BX, BC:BX, total pigment, and TDS were observed. Average gains per cycle were 13.15 and $4.06 \mathrm{mg}$ per $100 \mathrm{~g}$ fresh weight for $\mathrm{BC}$ and $\mathrm{BX}$ in HPHS, and 7.59 and $2.61 \mathrm{mg}$ per $100 \mathrm{~g}$ fresh weight for BC and BX in HPLS, respectively. Total pigment increased about $200 \%$ in both populations. Selection for low TDS was ineffective, while only a mild response was detected from selection for high TDS. Since betalain pigments are formed following glycosylation of cyclodopa and betalamic acid, sugar molecules are associated with pigment biosynthesis. Simultaneous selection for high pigment and low TDS may therefore be metabolically incompatible. Our findings demonstrate betalain pigment concentration responds favorably to recurrent selection: however. a concomitant reduction in TDS is not a feasible selection goal.
\end{abstract}

Betalain pigments extracted from red beet (Beta vulgaris) roots provide a natural alternative to synthetic red dyes. Betalains have been successfully used in commercial food coloring operations for a number of years (von Elbe et al., 1974), and continue to be an important source of red color in the food industry. Betalains are derivatives of betalamic acid and can be classified into two groups: the red-violet betacyanins (BC) and the yellow betaxanthins (BX). These differ by conjugation of a substituted aromatic nucleus to the 1,7-diazaheptamethinium chromophore, which is present in betacyanin.

The presence of dominant alleles at two closely linked loci ( $R$ and $Y$ ) condition production of betalain pigment in the beet plant (Keller, 1936). Wolyn and Gabelman (1989) demonstrated that three alleles at the $R$ locus determine the ratio of betacyanin to betaxanthin in the beet root and shoot. Despite the importance of these simplyinherited genes in pigment synthesis, several investigations suggest additional loci play a role in the quantity of betalain synthesized in the beet root (Watson and Gabelman, 1984, Wolyn and Gabelman, 1990). Populations of beet plants carrying dominant $R$ and $\mathrm{Y}$ alleles can be improved for pigment concentration. Watson and Gabelman (1984) observed small genotypic and phenotypic correlations between pigment concentration and sucrose content in red beet, suggesting that selection for high pigment and low sucrose was a feasible breeding goal. Wolyn and Gabelman (1989) confirmed this prediction by showing that betalain pigment responds to selection in a quantitative fashion in two beet populations. The main limitation to extraction and use of beet concen-

Received for publication 15 May 1995. Accepted for publication 6 Sept. 1995. This research supported by a grant from Aunt Nellies Farm Kitchens, Clyman, Wis, and Hatch project 3654, Wisconsin Agricultural Experiment Station, College of Agricultural and Life Sciences, Univ. of Wisconsin-Madison. We are indebted to J.F. Watson and D.J. Wolyn for developing the populations used in these investigations. The cost of publishing this paper was defrayed in part by the payment of page charges. Under postal regulations, this paper therefore must be hereby marked advertisement solely to indicate this fact.

Assistant professor.

${ }^{2}$ Research associate.

${ }^{3}$ Researcher.

${ }^{4}$ Professor emeritus. trates in the food industry is the relatively low concentration of betacyanin $(0.10 \%-0.18 \%$ of fresh weight) in beet root juice. Beet root juice contains high levels of sugars that dilute the commercial product (concentrated juice) substantially. Low concentrations of pigment necessitate the addition of large quantities of commercial product to foods to obtain sufficient coloring. Concentrating betacyanin in the root juice is a time and energy-consuming process for the food processor, often representing the most costly step in the extraction of betalains. Since sugar concentrations can be 80-200 times greater than pigment concentrations in the beet root, the commercial product would be significantly enhanced by lowering sugar (or solids) levels in the beet root through selection.

Betalain pigment is derived from betalamic acid following the cleavage of L-DOPA between the 4- and 5- positions (Clement et al. 1993; Fischer and Dreiding, 1972; Impellizzeri and Piattelli, 1972). The cleavage of L-DOPA results in two intermediates, 4,5secodopa and cyclodopa glucoside. The former intermediate is converted into betalamic acid, which in turn condenses with cyclodopa glucoside to form both betacyanin and betaxanthin. Glycosylation occurs both before and after the condensation reaction (Sciuto et al., 1972), and both pigment molecules contain glucose residues. The requirement of sugar in biosynthesis of the pigment molecule may play an important role in simultaneous selection for both high pigment and low solids.

In 1978, a recurrent selection program was initiated to increase pigment levels and decrease sugar [total dissolved solids (TDS)] levels in red beet. To gain knowledge about the response of beet to simultaneous selection for pigment and solids, divergent selection for both high and low TDS was practiced in conjunction with selection for high pigment. The two populations undergoing recurrent selection were named HPHS (high-pigment, high-solids) and HPLS (high-pigment, low-solids). Results from the first three cycles of selection demonstrated that pigment synthesis was highly responsive to selection (Wolyn and Gabelman, 1990). Pigment levels increased an average of $45 \%$ in three cycles. Simultaneous selection for low TDS (HPLS) was ineffective for the first three cycles. Selection for high TDS (HPHS) increased the relative mean values for TDS, although not significantly. 
Subsequent selection for high pigment and both high and low TDS were practiced in these populations until cycle 8. Remnant seed was then used to plant replicated field trials designed to evaluate response to selection. The objective of this investigation was to evaluate the response of HPHS and HPLS to eight cycles of recurrent half-sib family recurrent selection for pigment and TDS concentration.

\section{Materials and Methods}

Half-sib recurrent selection for high pigment and both high and low TDS was initiated in 1978 by J.F. Watson. The original population was formed from six open-pollinated cultivars chosen for high betalain pigment concentrations (Watson and Gabelman, 1984). Three U.S. cultivars (Garnet, Monoking Explorer, and Gladiator) and three European cultivars (Reglo, Rubia, and Boltardy) were used. Twenty beet roots of each cultivar were selected and planted in the greenhouse in the fall of 1978 where they were allowed to interpollinate. Equal quantities of seed from each plant were bulked and planted in 1979 in Madison, Wis. At harvest, 150 roots were chosen based on horticultural attributes, such as shape and size, and total pigment concentration. These roots were planted in the greenhouse and randomly intermated. Seed was harvested separately from each of 105 plants, producing 105 half-sib families.

Cycle O was planted in 1981. One-hundred five half-sib families and four parental cultivars (Reglo, Boltardy, Rubia, and Garnet) were planted in individual rows at the Walnut Street Garden in Madison. Ten roots from each row were harvested, based on the presence of desirable horticultural characteristics. After analysis for pigment and solids levels (using methods described below), 20 families with the highest BC concentration were chosen. Plants in the original (initiation) population were chosen for high betalain concentration, but plants in the cyclical selection process were selected for BC. Within selected families, seven roots with the highest pigment and TDS concentrations (HPHS) and highest pigment and the lowest TDS (HPLS) were chosen, resulting in 70 roots for seed production from each population. Samples of $50 \mathrm{~g}$ were excised from individual roots or bulked samples were excised consisting of five grams of each of ten roots per replicate from each family. Frozen samples were placed in a blender with water and homogenized for five min at high speed. Samples were further homogenized with additional water in a polytron. Fifteen $\mathrm{ml}$ aliquots of each sample were centrifuged for $45 \mathrm{~min}$ at $15,000 \mathrm{rpm}$. One $\mathrm{ml}$ of supernatant was diluted to a total volume of $100 \mathrm{ml}$ and spectrophotometric analyses were performed to determine total pigment and $\mathrm{BC}: \mathrm{BX}$ ratios. TDS was analyzed for a single drop of root exudate using a refractometer. $\mathrm{BC}$ and $\mathrm{BX}$ were calculated according to Wolyn and Gabelman (1990). Total pigment (TP) was represented as $\mathrm{BC}+\mathrm{BX}$. Data from bulked samples were used to calculate family means.

Cycles 1,2,3, and $4(1982,1983,1984$, and 1990) were developed in the following manner. HPHS and HPLS populations were planted in two replicates of 70 half-sib families each. Twenty roots were selected from each family (10 from each of two replications) and analyzed for pigment and TDS. The seven roots with highest pigment and TDS concentrations for HPHS and highest pigment and lowest TDS for HPLS were chosen within each of the 10 best families producing 70 halfsib families for seed production. Cycles 5,6, and 7 (1991, 1992, and 1993) followed the same procedure except the HPLS population was selected for both high BC and low BX in addition to low TDS.

Seeds were randomly-selected from $\mathrm{C} 1$ through $\mathrm{C} 8$ in both HPHS and HPLS and sown at a density of 35 seeds per meter in 4-m single-row plots at two locations (Arlington Experiment Station in Arlington, Wis., and Walnut Street Garden in Madison) during 1994. Cycle entries were arranged in three replications of a randomized complete block design. Root tissue was excised from a sample of 20 beets in each plot and combined for pigment and TDS analyses. Pigment and TDS analyses were performed as described above (Wolyn and Gabelman, 1990). Data were analyzed using the ANOVA procedure of SAS (Cary, N.C).

\section{Results and Discussion}

The location main effect was highly significant for TDS in both HPHS and HPLS and for BC, BX, and TP in HPLS (Table 1). Higher mean pigment values were measured at Madison than at Arlington, however few relative differences among cycles were consistent for the two locations since the location $\times$ cycle interaction was not significant for any of the traits measured except for TDS in HPHS. The analysis of variance revealed highly significant differences among cycles for BC, BX, BC:BX, TP, and TDS for HPHS. Only BC, $\mathrm{BX}$, and TP were significantly different among cycles for HPLS.

Table 1. Mean squares from the analysis of variance for response to eight cycles of recurrent halfsib selection for high pigment and both high and low total dissolved solids.

\begin{tabular}{|c|c|c|c|c|c|c|}
\hline $\begin{array}{l}\text { Source of } \\
\text { variation }\end{array}$ & df & Betacyanin & Betaxanthin & Total pigment & $\mathrm{BC}: \mathrm{BX}^{\mathrm{z}}$ & $\operatorname{TDS}^{\mathrm{Y}}$ \\
\hline \multicolumn{7}{|c|}{$H P H S$} \\
\hline Location & 1 & 53.36 & 1.20 & 38.47 & 0.07 & $20.02 * * *$ \\
\hline Replication & 2 & 383.07 & 86.05 & 583.07 & $0.46^{*}$ & 3.56 *** \\
\hline Cycle & 7 & $6754.38 * * *$ & $768.51 * * *$ & $11590.37 * * *$ & $0.69 * * *$ & $1.39^{* * *}$ \\
\hline \multicolumn{7}{|c|}{$H P H S$} \\
\hline Location & 1 & $3525.01 * * *$ & $599.32 * * *$ & $7032.52 * * *$ & 0.27 & $5.64 * * *$ \\
\hline Replication & 2 & 43.13 & 6.33 & 49.41 & 0.15 & 0.31 \\
\hline Cycle & 7 & $3197.32 * * *$ & $423.27 * * *$ & $5788.84 * * *$ & 0.36 & 0.35 \\
\hline Location*cycle & 7 & 64.97 & 67.58 & 191.47 & 0.42 & 0.31 \\
\hline
\end{tabular}

${ }^{2} \mathrm{BC}: \mathrm{BX}=$ betacyanine : betaxanthine ratio.

${ }^{\mathrm{y}} \mathrm{TDS}=$ total dissolved solids.

$*, * *, * *$ Significant at $P<0.10,0.05$, and 0.01 levels, respectively. 

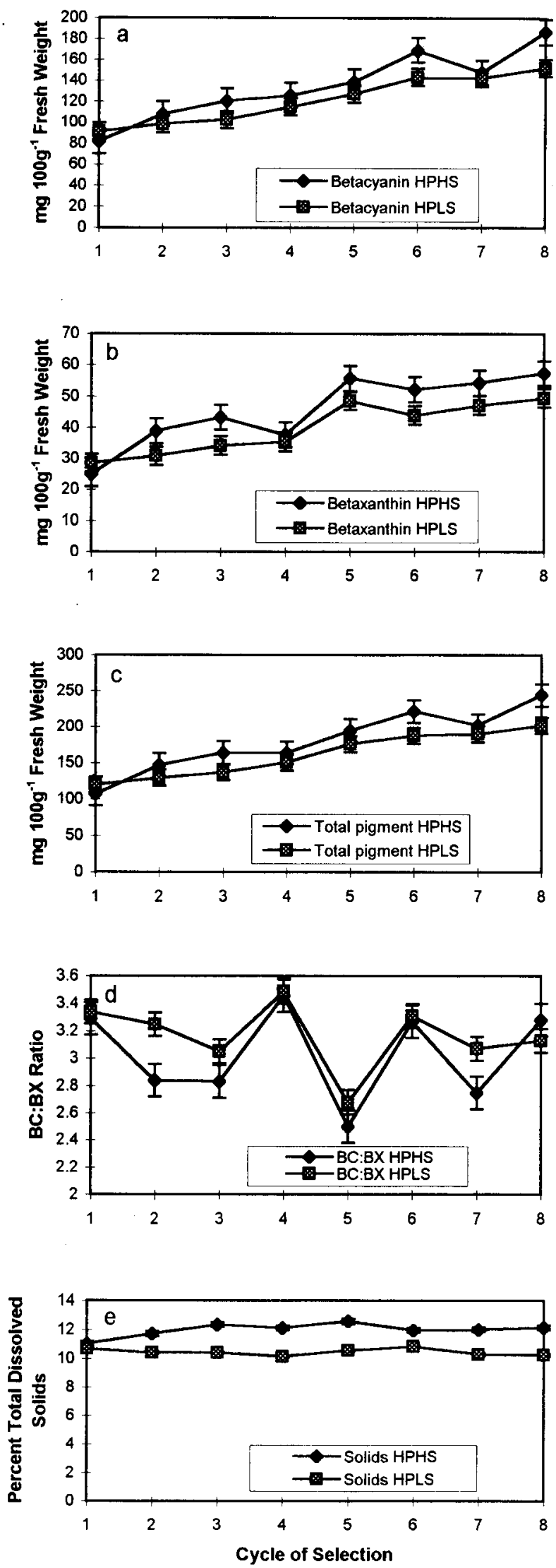

Fig. 1. Response of two populations (HPHS and HPLS) to recurrent half-sib selection for high pigment and both high and low total dissolved solids. A-E represent response of betacyanin $(\mathrm{BC})$, betaxanthin $(\mathrm{BX})$, total pigment, $\mathrm{BC}$ : BX ratio, and total dissolved solids, respectively. Vertical bars indicate standard errors.
Changes over cycles for $\mathrm{BC}, \mathrm{BX}$, and $\mathrm{TP}$ proceeded in a linear fashion (Fig. 1). Mean BC increased from 80 to $180 \mathrm{mg}$ per $100 \mathrm{~g}$ fresh weight in HPHS over the eight cycles of selection. Mean BC increased from 90 to $140 \mathrm{mg}$ per $100 \mathrm{~g}$ fresh weight in HPLS over the eight cycles of selection, a significant but more gradual increase. The average gains per cycle for BC were 13.15 and 7.54 $\mathrm{mg}$ per $100 \mathrm{~g}$ fresh weight in HPHS and HPLS, respectively (Table 2). Mean BX increased from 25 to $55 \mathrm{mg}$ per $100 \mathrm{~g}$ fresh weight in HPHS during the selection process, while an increase from 28 to $48 \mathrm{mg}$ per $100 \mathrm{~g}$ fresh weight was observed in HPLS. The average gains per cycle for $\mathrm{BX}$ were 4.06 and $2.61 \mathrm{mg}$ per $100 \mathrm{~g}$ fresh weight in HPHS and HPLS, respectively. TP increased from 112 to $240 \mathrm{mg}$ and from 115 to $180 \mathrm{mg}$ per $100 \mathrm{~g}$ fresh weight, in HPHS and HPLS, respectively. These gains represent a per cycle increase of 17.21 and $10.20 \mathrm{mg}$ per $100 \mathrm{~g}$ fresh weight in HPHS and HPLS, respectively. Higher values of $\mathrm{BC}, \mathrm{BX}$, and $\mathrm{TP}$ were measured in HPHS than in HPLS for most cycles of selection although the two populations closely parallel each other in terms of their selection response. This finding suggests selection for high TP in combination with high TDS is more effective in increasing TP than when concurrently selecting for low TDS. Wolyn and Gabelman ( 1990) described a similar response for the first three cycles of selection in these populations.

Gain per cycle for BC and BX was about twice as great in HPHS than in HPLS (Table 2). Gain per cycle for TP was also substantially higher $(70 \%)$ in HPHS than in HPLS. These data suggest simultaneous selection for high pigment and high TDS is more effective than simultaneous selection for high pigment and low TDS. Wolyn and Gabelman (1990) measured a $22.2 \%$ gain per cycle for TP in HPHS and an $18.4 \%$ gain per cycle in HPLS after three cycles of recurrent selection. The data reported herein demonstrate the level of gain reported by Wolyn and Gabelman was not sustained throughout the eight cycles of selection, however steady gains were observed for all three pigment traits. Since BC comprises a larger portion of TP (about 80\%) than BX, it is expected that larger gains from selection were measured for $\mathrm{BC}$ than for $\mathrm{BX}$. Our data show that difference to be approximately 4 fold when comparing $\mathrm{BC}$ to $\mathrm{BX}$.

The $\mathrm{BC}: \mathrm{BX}$ ratio exhibited large but non-directional fluctuations across cycles of selection. BC:BX ranged from 2.5 to 3.5 but remained at about 3.1 for most of the cycles of selection. An increase and a large decrease in $\mathrm{BC}: \mathrm{BX}$ were observed in cycles 4 and 5 , respectively, contributing to the significance of this term in the analysis of variance. Overall fluctuation in this ratio did not appear to be correlated with responses measured for BC, BX, and TP. This finding demonstrates the spectral quality of the pigment was maintained throughout the recurrent selection program. Wolyn and Gabelman (1990) described an increase in BC:BX in both HPHS and HPLS over the first three cycles of selection. When all eight cycles were evaluated in controlled environments, a decrease in BC:BX was observed over the first three cycles of selection (Fig. 1). This finding demonstrates the differences in evaluating selected cycles in controlled environments versus evaluation of cycles sequentially as they are grown and evaluated in successive years. Our data do not support the finding that $\mathrm{BC}: \mathrm{BX}$ changes directionally in response to selection, suggesting concurrent increases in both pigments occurred during recurrent selection in both populations.

TDS increased from $119 \mathrm{Z} 0$ to $12.2 \%$ in HPHS over eight cycles of selection, however a level of $12.2 \%$ TDS was reached by cycle 3 and did not change appreciably in the latter five cycles. A nonsignificant decrease was measured for TDS in HPLS from $10.7 \%$. to $10.3 \%$. Despite the effectiveness of the initial separation 
Table 2. Average gain per cycle of selection for eight cycles of recurrent half-sib selection for high pigment and high and low total dissolved solids.

\begin{tabular}{lccc}
\hline \hline Population & Betacycanine & Betaxanthine & Total pigment \\
\hline & \multicolumn{3}{c}{$m g / 100 \mathrm{~g}$ fresh $w t$} \\
HPHS & 13.15 & 4.06 & 17.21 \\
HPLS & 7.59 & 2.61 & 10.20 \\
\hline
\end{tabular}

of the populations into HPHS and HPLS at cycle 1, little significant change in TDS was observed beyond cycle 3 in HPHS and HPLS. This finding suggests simultaneous selection for both high pigment and either high or low TDS is largely ineffective. Wolyn and Gabelman ( 1990) measured no significant change in TDS in either HPHS or HPLS; however, a trend toward increased TDS was observed in HPHS. This trend was detected in our study; however, as previously mentioned, changes in TDS were not observed beyond cycle 3 for HPHS.

The lack of significant directional selection response in TDS in both populations may be caused by several factors. Measurement of TDS in red beet via refractometric analysis is inherently difficult because colored solutions obscure precise data collection. As coloration of solutions increased due to selection, difficulties in refractometric analysis were amplified. Despite these difficulties, it is possible simultaneous selection for high TP and low TDS is limited by the need for sugar molecules in the synthesis of both BC and BX. Since both BC and BX are formed following glycosylation of the condensation reaction between cyclodopa and betalamic acid, the failure to decrease TDS may be ascribed to the need for sugar molecules in betalain biosynthesis. This being the case, larger increases in TDS in the HPHS population might be expected than were realized in this study. Because the error among plants within a cycle for TDS was small in this experiment, we suggest the lack of response to selection maybe associated with the directly competing objectives of selecting for high TP and low TDS.

\section{Literature Cited}

Clement, J. S., T.J. Mabry, H. Wyler, and A.S. Dreiding. 1992. Chemical review and evolutionary significance of the betalains. In Evolution and Systematic of the Caryophyllales. H.D. Behnke and T.J. Mabry (eds.). Springer Verlag.

Fischer, N. and A.S. Dreiding. 1972. Biosynthesis of betalaines. On the cleavage of the aromatic ring during the enzymatic transformation of dopa into betalamic acid. Helv. Chim. Acta 55:649-658.

Impellizzeri, G. and M. Piatelli. 1972. Biosynthesis of betalains: formation of indicaxanthin in Opuntia ficus-indica fruits. Photochemistry 11:2499-2502.

Keller, W. 1936. Inheritance of some major color types in beets. J. Agr. Res. 52:27-38.

Knuthsen, P. 1981. Investigations on beetroot colors for the purpose of regulation. Z Lebensm Unters Forsch 172: 195-200.

Sciuto, S., G.P. Oriente, and M. Piatelli. 1972. Betalain glucosylation in Opuntia dillenii. Phytochem. 11:2259-2262.

von Elbe J. H., J.H. Pasch, and J.P. Adams. 1974. Betalains as food colorants. Proc. IV Intl. Congr. Food Sci. and Technol. 1:485492

Watson, J.F. and W.H. Gabelman. 1984. Genetic analysis of betacyanin, betaxanthin, and sucrose concentrations in roots of table beet. J. Amer. Soc. Hort. Sci. 109:386-391.

Wolyn, D.J. and W.H. Gabelman. 1989. Inheritance of root and petiole pigmentation in red table beet. J. Hered. 80:33-38.

Wolyn, D.J. and W.H. Gabelman. 1990. Selection for betalain pigment concentrations and total dissolved solids in red table beets. J. Amer. Sot. Hort. Sci. 115(1):165-169. 